\title{
Impact of Mobility on Spectrum Sensing in Cognitive Radio Networks
}

\author{
Alexander W. Min and Kang G. Shin \\ Real-Time Computing Laboratory \\ Department of Electrical Engineering and Computer Science \\ The University of Michigan, Ann Arbor, MI 48109-2121, USA
}

\{alexmin, kgshin\}@eecs.umich.edu

\begin{abstract}
In cognitive radio networks (CRNs), spectrum sensing is key to opportunistic spectrum access while preventing any unacceptable interference to primary users' communications. Although cognitive radios function as spectrum sensors and move around, most, if not all, of existing approaches assume stationary spectrum sensors, thus providing inaccurate sensing results. As part of our effort to solve/alleviate this problem, we consider the impact of sensor mobility on spectrum sensing performance in a joint optimization framework for sensor cooperation and sensing scheduling. We show that sensor mobility increases spatio-temporal diversity in received primary signal strengths, and thus, improves the sensing performance. This is intuitively plausible, but have not been tested previously. Based on this observation, we propose a sensing strategy that minimizes the sensing overhead by finding an optimal combination of the number of sensors to cooperate and the number of times spectrum sensing must be scheduled. This result provides a useful insight to understand the spectrum sensing and its coupling with sensor mobility.
\end{abstract}

\section{Categories and Subject Descriptors}

C.2.1 [Computer-Communication Networks]: Network Architecture and Design-Wireless communication

\section{General Terms}

Algorithms, Performance, Theory

\section{Keywords}

Cognitive radios, Sensor mobility, Spectrum sensing

\section{INTRODUCTION}

In cognitive radio networks (CRNs), spectrum sensing must meet the strict detectability requirements set by the FCC to protect primary users' communications from excessive interference caused by secondary CR devices. To meet these requirements, cooperative sensing $[12,19]$ and sensing

Permission to make digital or hard copies of all or part of this work for personal or classroom use is granted without fee provided that copies are not made or distributed for profit or commercial advantage and that copies bear this notice and the full citation on the first page. To copy otherwise, to republish, to post on servers or to redistribute to lists, requires prior specific permission and/or a fee.

CoRoNet'09, September 21, 2009, Beijing, China

Copyright 2009 ACM 978-1-60558-738-7/09/09 ...\$10.00. scheduling $[8,11]$ have been studied as efficient means to improve the sensing performance by exploiting spatio-temporal diversity in received signal strengths (RSSs). In [11], we proposed a sensing framework that minimizes the sensing-time while meeting the detection requirements by jointly optimizing sensor selection and sensing scheduling. An interesting observation made there is that when sensors are stationary as in 802.22 WRANs, the measured RSSs at each sensor are pseudo time-invariant, depending on their geographical location, thus limiting the performance gain from sensing scheduling.

Mobility is one of the most important factors in wireless systems because it affects numerous network characteristics, such as network capacity [6], connectivity [14], coverage [9], routing [10], etc. It is also an inherent feature to support various types of wireless services in CRNs. While the 802.22 Working Group considered only stationary sensors (i.e., CPEs) in the initial standard draft, recently, they adopted an amendment for the operation of portable devices [18]. Despite its importance, however, mobility is still largely unexplored in the context of dynamic spectrum access. Allowing sensor mobility in CRNs will introduce numerous challenges, making it necessary to revisit current system design and protocols, such as mechanisms for spectrum sensing, interference management and routing.

As a first step to understand the impact of mobility in CRNs, we study the performance of spectrum sensing with mobile sensors via a theoretical study. In particular, we show that, when sensing is scheduled multiple times, sensor mobility can yield a significant performance gain by exploiting spatio-temporal diversity in received primary signal strengths. This is in sharp contrast to the case of stationary sensors where the benefit to be gained from scheduling sensing is marginal. Our theoretical analysis indicates that the contribution of sensing scheduling to the performance improvement increases as the speed of mobile sensor increases, which raises an interesting question: how to establish a balance between the number of sensors to use and the number of times to sense? To address this question, we derive an optimal combination of these two design parameters that minimizes the overall sensing overhead. To our best knowledge, this is the first study to examine the impact of sensor mobility on the performance of spectrum sensing.

\section{RELATED WORK}

Distributed sensing has been recognized as a viable means to improve the sensing performance while meeting the stringent incumbent detection requirements set by the FCC. Its performance has been studied extensively, including tradeoffs in spectrum sensing [8], detectability in fading environ- 


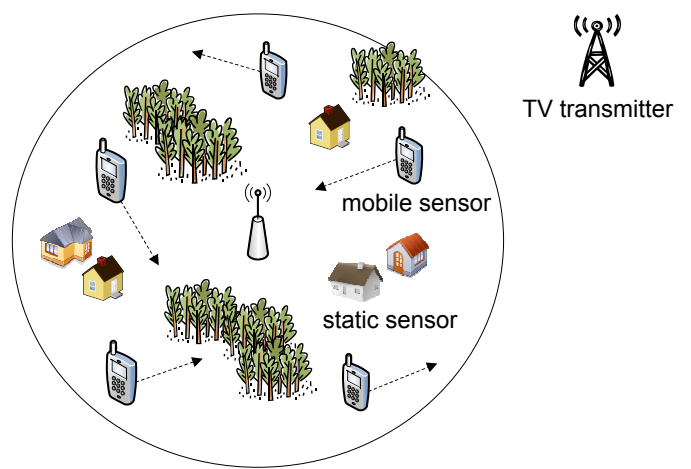

Figure 1: An example of the 802.22 WRAN with a large-scale primary user, i.e., TV transmitter, and static and mobile sensors.

ments [12], and impact on transport layers [3]. However, the sensor cooperation gain has been reported to degrade as the shadow fading correlation among collaborating sensors increases [12]. For example, Ghasemi and Sousa [5] derived an asymptotic bound of detection performance in a correlated log-normal shadowing environment. They showed that the performance improvement by having an infinite number of correlated sensors is bounded depending on the degree of correlation. Along the same line as in [5], Visotsky et al. [19] proved that by increasing the number of sensors with i.i.d. shadow fading, both false-alarm and missdetection probabilities can be made arbitrarily small. To mitigate this adverse impact of shadow correlation, Selén et al. [15] proposed heuristic sensor selection algorithms to find independent sensors for distributed sensing.

While cooperative sensing aims to exploit sensor location diversity (in space domain), sensing scheduling has recently been studied as an alternative means to improve the sensing performance (in time domain) [2,11]. However, Min and Shin [11] demonstrated that sensing scheduling with static sensors does not make a considerable performance gain because of limited diversity in RSSs due to their fixed geographical location. In this paper, as a generalization of the previously-proposed joint optimization framework for distributed sensing [11], we consider mobile sensors and study the impact of sensor mobility on the performance of spectrum sensing and corresponding design tradeoffs between cooperation and scheduling.

\section{SYSTEM MODEL}

A CRN consists of a large-scale primary (i.e., TV) transmitter and an infrastructure-based secondary network with static and mobile sensors, as shown in Fig. 1. In an IEEE 802.22 WRAN, a base station coordinates distributed spectrum sensing within a cell by directing a set of static and/or mobile sensors to sense a target channel. We assume that the mobile sensors move independently of each other within the cell, and thus, there is no correlation among the sensors. ${ }^{1}$ The sensors will lose their connectivity to the base station as they move outside the cell boundary; otherwise, they might cause an excessive interference to the primary receivers.

\footnotetext{
${ }^{1}$ This is a reasonable assumption because decorrelation distance is in the range of $120-200(\mathrm{~m})$ in suburban areas [1], whereas the typical cell radius is $33 \mathrm{~km}$ [4].
}

To detect the existence of a primary signal, a simple energy detector is used to focus on characterization of the achievable performance gain using mobile sensors. The output of the energy detector, denoted by the test statistic $T$, is basically an estimate of the received primary signal plus noise power. The test statistic at sensor $n$ can be approximated as a Gaussian distribution using the Central Limit Theorem (CLT) [16]:

$$
T_{n} \sim \begin{cases}\mathcal{N}\left(N_{o}, \frac{N_{o}^{2}}{M_{s}}\right) & \text { under } \mathcal{H}_{0} \text { (white space) } \\ \mathcal{N}\left(P_{n}+N_{o}, \frac{\left(P_{n}+N_{o}\right)^{2}}{M_{s}}\right) & \text { under } \mathcal{H}_{1} \text { (occupied) }\end{cases}
$$

where $P_{n}$ is the received primary signal strength, $N_{o}$ the noise power, and $M_{s}$ the number of signal samples during the sensing period $T_{S}$ (e.g., $1 \mathrm{~ms}$ ). We assume that the signal is sampled at the Nyquist rate, i.e., $6 \mathrm{MHz}$, so it takes $1 \mathrm{~ms}$ to obtain $M_{s}=6 \times 10^{3}$ samples.

The received primary signal strength at sensor $n$ can be expressed as:

$$
P_{n}=P_{R} \cdot e^{Y_{n}}
$$

where $P_{R}$ is the average received signal strength within a cell, ${ }^{2}$ and $e^{Y_{n}}$, where $Y \sim \mathcal{N}\left(0, \sigma^{2}\right)$, is the channel gain between the primary transmitter and sensor $n$ due to $\log$ normal shadowing. The log-normal shadow fading is often characterized by the dB-spread $\sigma_{d B}$, which has the following relationship $\sigma=0.1 \log _{e}(10) \sigma_{d B}$. Note that the effect of multi-path fading is negligible in a DTV channel because of its wide bandwidth [16].

\section{SPECTRUM SENSING WITH MOBILE SENSORS}

In this section, we first characterize the temporal correlation in test statistics from a single mobile sensor. We then formulate the primary signal detection as a hypothesis testing problem with multiple mobile sensors, each sensing/sampling the spectrum multiple times.

\subsection{Correlated Measurements from a Single Mobile Sensor}

Let's first consider the results from a single mobile sensor. Let $\mathbf{x}_{n}=\left[T_{n 1}, \ldots, T_{n M}\right]^{T}$ denote the test statistics (i.e., energy detector output) measured at a mobile sensor $n$, as a result of sensing $M$ times at a discrete time interval $\Delta t$. Since the test statistics $T$ is Gaussian-distributed, the measurement vector $\mathbf{x}_{n}$ is $M$-variate Gaussian under both hypotheses:

$$
\mathbf{x}_{n} \sim \begin{cases}\mathcal{N}\left(\mu_{0} \times \mathbf{1}, \boldsymbol{\Sigma}_{n}\right) & \text { under } \mathcal{H}_{0} \\ \mathcal{N}\left(\mu_{1} \times \mathbf{1}, \boldsymbol{\Sigma}_{n}\right) & \text { under } \mathcal{H}_{1}\end{cases}
$$

where $\mu_{0}=\left(P_{R, 0}+N_{o}\right) \times \mathbf{1}$ and $\mu_{1}=\left(P_{R, 1}+N_{o}\right) \times \mathbf{1} . P_{R, 0}$ and $P_{R, 1}$ are the average received primary signal strengths in the cell under $\mathcal{H}_{0}$ and $\mathcal{H}_{1}$, respectively, and $\mathbf{1}=[1, \ldots, 1]^{T}$. Without loss of generality, we can assume $P_{R, 0} \neq 0 \ll P_{R, 1}$ to have a common covariance matrix $\boldsymbol{\Sigma}_{n}$ under both hypotheses. $^{3}$

The sequence of measured RSSs (i.e., $\mathbf{x}_{n}$ ) from a mobile sensor will be correlated corresponding to their geographical separation between the sensing events due to the correlated

\footnotetext{
${ }^{2}$ We assume that the path-loss effect is approximately same within the cell because the distance to the primary transmitter is relatively large compared to the cell radius.

${ }^{3}$ With uncommon covariance matrices, there is no closedform expression for the detection probabilities [13].
} 

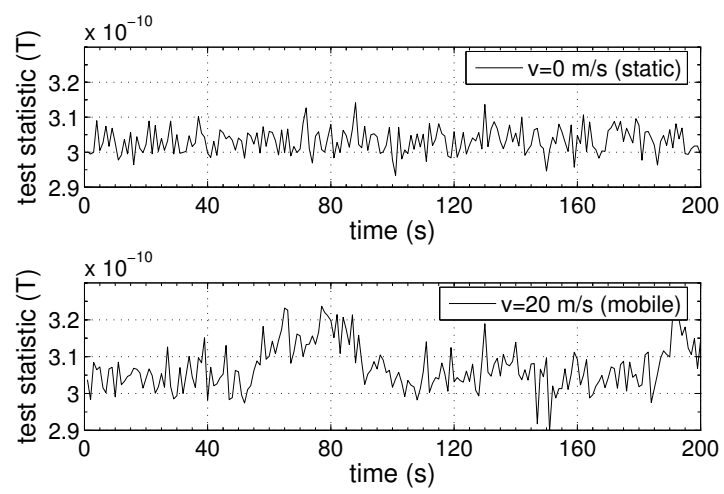

Figure 2: Example time-sequences of energy detector outputs ( $\mathbf{T}$ ) under shadow fading (with $\sigma_{d B}=$ $5.5 \mathrm{~dB}$ ) with static (the upper figure) and mobile (the lower figure) sensors. The parameters used are $P_{R, 1}=-116 \mathrm{dBm}, N_{o}=-95.2 \mathrm{dBm}$, and $T_{S}=1 \mathrm{~ms}$, as typically assumed in IEEE 802.22.

shadowing (i.e., $e^{Y_{n}}$ in Eq. (1)). The temporal correlation in $\mathbf{x}_{n}$ can be captured by analyzing the covariance matrix $\boldsymbol{\Sigma}_{n}$ in Eq. (2). First, the measurement error of the energy detector (i.e., variance of the test statistic $T$ ) can be approximated as $\frac{\left(P_{n}+N_{o}\right)^{2}}{M_{s}} \approx \frac{N_{o}^{2}}{M_{s}}+\frac{2 N_{o} P_{n}}{M_{s}} \forall n$. This is based on the fact that in a very low SNR environment such as $-20 \mathrm{~dB}$, the received primary signal strength $P_{n}$ may be significantly lower than the noise power, i.e., $P_{n} \ll N_{o}$ [11]. Then, assuming the Gudmundson's exponential decaying model [7], the covariance matrix $\boldsymbol{\Sigma}_{n}$ is a symmetric Toeplitz matrix as:

$$
\boldsymbol{\Sigma}_{n} \triangleq \frac{N_{o}^{2}}{M_{s}} \mathbf{I}+\frac{2 N_{o} P_{R}}{M_{s}}\left[\begin{array}{cccc}
1 & \rho_{n} & \cdots & \rho_{n}^{M-1} \\
\rho_{n} & 1 & \cdots & \rho_{n}^{M-2} \\
\vdots & \vdots & \ddots & \vdots \\
\rho_{n}^{M-1} & \rho_{n}^{M-2} & \cdots & 1
\end{array}\right]
$$

where

$$
\rho_{n}=e^{-\Delta d_{n} / d_{\text {corr }}} \approx e^{-v_{n} \Delta t / d_{\text {corr }}},
$$

where $d_{\text {corr }}$ is the decorrelation distance of shadow fading, and $\Delta d_{n}$ is the geographical separation (i.e., Euclidean distance) between consecutive sensing events. The approximation in Eq. (4) is based on the assumption that the mobile nodes do not change their direction in the middle of consecutive sensing events; this is a reasonable assumption due to short sensing intervals, e.g., $\Delta t<1 \mathrm{~s}$, in practice. We assume that the sensor speed is fixed at $v_{n} \mathrm{~m} / \mathrm{s}$, and thus, the sensor travels the same distance during every sensing interval.

Fig. 2 compares the time-sequences of test statistics $T$ of a static sensor (the upper figure) and of a mobile sensor, which traverses a straight line within a cell area with $v=20 \mathrm{~m} / \mathrm{s}$ (the lower figure). Note that a static sensor experiences (pseudo) time-invariant RSSs (except for measurement errors) due to lack of mobility, and the average RSS depends on its geographical location [11]. By contrast, the lower figure clearly shows that the mobile sensor exhibits temporal variations in $T$ due to spatially-correlated shadow fading.

\subsection{Hypothesis Testing}

We now formulate the primary signal detection with multiple mobile sensors as a hypothesis testing problem. Let $\mathbf{x}=\left[\mathbf{x}_{1}^{T}, \ldots, \mathbf{x}_{N}^{T}\right]^{T}$ denote the $N \times M$ RSS measurement matrix collected from $N$ mobile sensors, where each sensor senses the spectrum $M$ times. Then, the primary signal detection problem can be cast into the following hypothesis testing problem:

$$
\begin{cases}\mathcal{H}_{0}: \mathbf{x} \sim \mathcal{N}\left(\mu_{0}, \boldsymbol{\Sigma}\right) & \text { (white space) } \\ \mathcal{H}_{1}: \mathbf{x} \sim \mathcal{N}\left(\mu_{1}, \boldsymbol{\Sigma}\right) & \text { (occupied) }\end{cases}
$$

where the common covariance matrix $\boldsymbol{\Sigma}$ is given as:

$$
\boldsymbol{\Sigma} \triangleq\left[\begin{array}{cccc}
\boldsymbol{\Sigma}_{1} & 0 & \cdots & 0 \\
0 & \boldsymbol{\Sigma}_{2} & \cdots & 0 \\
\vdots & \vdots & \ddots & \vdots \\
0 & 0 & \cdots & \boldsymbol{\Sigma}_{N}
\end{array}\right]_{N M \times N M}
$$

Recall that sensors move independently within a large cell area (e.g., of radius up to $100 \mathrm{~km}$ ), and thus it is reasonable to assume that the correlation between samples collected from different sensors is negligible, i.e., $\Sigma_{i, j} \approx 0 \forall i, j$ s.t. $\lfloor(i-1) / M\rfloor \neq\lfloor(j-1) / M\rfloor$ in Eq. (6).

\section{THEORETICAL ANALYSIS}

We now analyze the theoretical sensing performance with mobile sensors. In order to quantify the achievable performance gain from sensor mobility, we consider an optimal data-fusion rule (i.e., a likelihood ratio test) for primary signal detection.

\subsection{Spatio-Temporal Spectrum Sensing}

We study the sensing performance in joint sensor cooperation and sensing scheduling. Assuming complete knowledge of fading channels, the likelihood ratio can be expressed as:

$$
\begin{aligned}
& \lambda(\mathbf{x}) \triangleq \frac{f\left(\mathbf{x} \mid \mathcal{H}_{1}\right)}{f\left(\mathbf{x} \mid \mathcal{H}_{0}\right)} \\
& =\exp \left[\left(\mu_{1}-\mu_{0}\right)^{T} \boldsymbol{\Sigma}^{-1} \mathbf{x}+\frac{1}{2}\left(\mu_{0}-\mu_{1}\right)^{T} \boldsymbol{\Sigma}^{-1}\left(\mu_{0}-\mu_{1}\right)\right],
\end{aligned}
$$

where $\mathbf{x}$ is the $N \times M$ matrix consists of sensing samples collected at the base station, $\boldsymbol{\Sigma}$ is the common covariance matrix in Eq. (6). The average received primary signal strengths and their pdfs under both hypotheses are denoted by $\mu_{i}$ and $f\left(\cdot \mid \mathcal{H}_{i}\right), i \in\{0,1\}$, respectively.

The optimal data-fusion is a log-likelihood ratio test (LRT) [13] with the following threshold-based decision rule: ${ }^{4}$

$$
\boldsymbol{\Lambda}(\mathbf{x}) \triangleq \log \lambda(\mathbf{x})=\mathbf{w}^{T}\left(\mathbf{x}-\mathbf{x}_{0}\right) \underset{\mathcal{H}_{0}}{\stackrel{\mathcal{H}_{1}}{\gtrless}} \eta
$$

where $\mathbf{w} \triangleq \boldsymbol{\Sigma}^{-1}\left(\mu_{1}-\mu_{0}\right)$ and $\mathbf{x}_{0} \triangleq \frac{1}{2}\left(\mu_{1}+\mu_{0}\right)$.

Then, the probability of false alarm with the decision threshold $\eta \in \mathbb{R}$ is given as:

$$
\begin{aligned}
P_{F A} & \triangleq \operatorname{Prob}\left(\boldsymbol{\Lambda}(\mathbf{x})>\eta \mid \mathcal{H}_{0}\right) \\
& =Q\left(\frac{\eta-\frac{1}{2} \mathbf{w}^{T} \mathbf{\Sigma} \mathbf{w}}{\sqrt{\mathbf{w}^{T} \mathbf{\Sigma} \mathbf{w}}}\right),
\end{aligned}
$$

where $Q(x)=\frac{1}{\sqrt{2 \pi}} \int_{x}^{\infty} e^{-t^{2} / 2} \mathrm{~d} t$. Using Eq. (8), the decision threshold $\eta$ subject to the desired $P_{F A}$ can be derived as:

$$
\eta=\sqrt{\mathbf{w}^{T} \boldsymbol{\Sigma} \mathbf{w}} \cdot Q^{-1}\left(P_{F A}\right)-\frac{1}{2} \mathbf{w}^{T} \mathbf{\Sigma} \mathbf{w} .
$$

\footnotetext{
${ }^{4}$ In practice, equal gain combining (EGC) achieves nearoptimal performance without estimating channel gains [17].
} 


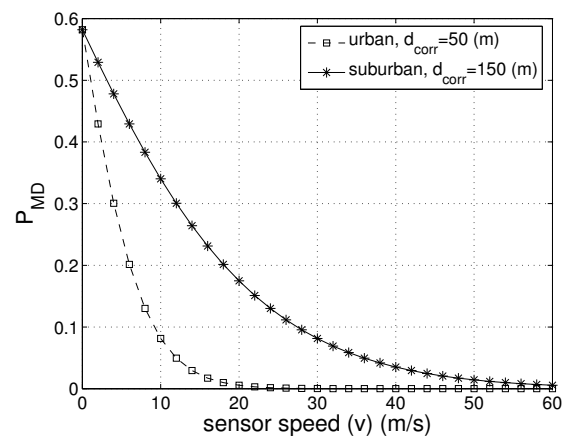

(a) Effect of sensor speed

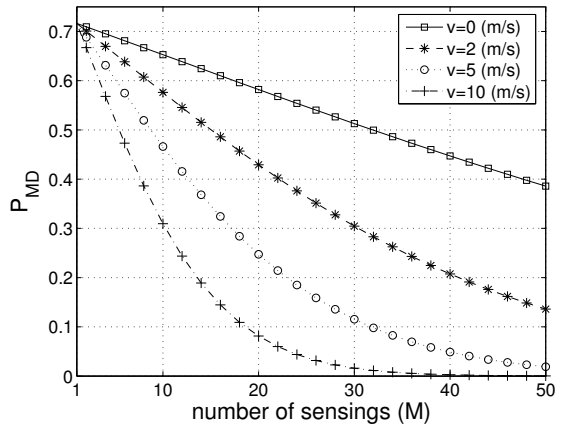

(b) Effect of sensing scheduling

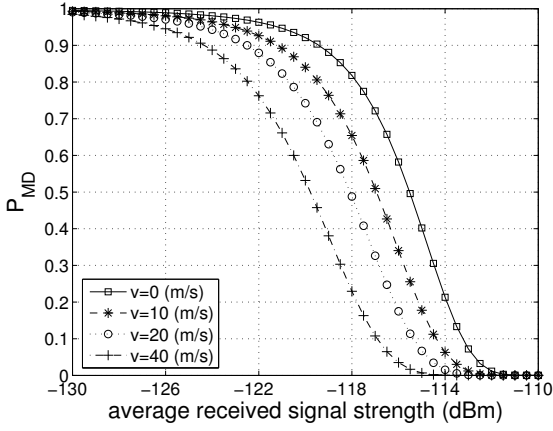

(c) Effect of average RSS

Figure 3: Performance of spatio-temporal sensing with mobile sensors. The figures show that sensor mobility (a) reduces the miss-detection probability, (b) increases sensing scheduling gain, and (c) better detects weak primary signals. The parameters used are $N=1, M=20, P_{R}=-116 \mathbf{d B m}, N_{o}=-95.2 \mathbf{d B m}, T_{S}=1 \mathbf{m s}, \Delta t=1 \mathrm{~s}$, $d_{\text {corr }}=150 \mathrm{~m}$, and $P_{F A}=0.001$, unless specified otherwise.

Based on Eqs. (8) and (9), the probability of miss detection, $P_{M D}$, is given as:

$$
\begin{aligned}
P_{M D} & \triangleq \operatorname{Prob}\left(\boldsymbol{\Lambda}(\mathbf{x})<\eta \mid \mathcal{H}_{1}\right) \\
& =1-Q\left(Q^{-1}\left(P_{F A}\right)-\frac{\left(\mu_{1}-\mu_{0}\right)}{2} \sqrt{\mathbf{1}^{T} \mathbf{\Sigma}^{-1} \mathbf{1}}\right),
\end{aligned}
$$

where $\boldsymbol{\Sigma}^{-1}=\operatorname{diag}\left\{\boldsymbol{\Sigma}_{1}^{-1}, \ldots, \boldsymbol{\Sigma}_{N}^{-1}\right\}$, and each element $\boldsymbol{\Sigma}_{n}^{-1}$ can be derived as a sum of identity and tri-diagonal matrices:

$$
\begin{gathered}
\boldsymbol{\Sigma}_{n}^{-1}=\frac{M_{s}}{N_{o}^{2}} \mathbf{I}+ \\
\frac{M_{s}}{2 N_{o} P_{R}\left(1-\rho_{n}^{2}\right)}\left[\begin{array}{ccccc}
1 & -\rho_{n} & 0 & \cdots & 0 \\
-\rho_{n} & 1+\rho_{n}^{2} & -\rho_{n} & \cdots & 0 \\
\vdots & \ddots & \ddots & \ddots & \vdots \\
0 & \cdots & -\rho_{n} & 1+\rho_{n}^{2} & -\rho_{n} \\
0 & \cdots & 0 & -\rho_{n} & 1
\end{array}\right] .
\end{gathered}
$$

We examine the behavior of $P_{M D}$ with $P_{F A}$ fixed. Eq. (10) indicates that the achievable $P_{M D}$ is determined by the covariance matrix $\boldsymbol{\Sigma}$ with the other parameters predefined. The term that includes $\boldsymbol{\Sigma}$ in Eq. (10), can be expressed as:

$$
\begin{aligned}
& \sqrt{\mathbf{1}^{T} \boldsymbol{\Sigma}^{-1} \mathbf{1}}=\sqrt{\sum_{n} \mathbf{1}^{T} \boldsymbol{\Sigma}_{n}^{-1} \mathbf{1}} \\
& =\sqrt{\frac{N M}{\sigma_{n}^{2}}+\frac{M_{s}}{2 N_{o} P_{R}} \sum_{n} \frac{\left(1-\rho_{n}\right) M+2 \rho_{n}}{1+\rho_{n}}},
\end{aligned}
$$

where $\sigma_{n}^{2}$ is the variance of the test statistic, i.e., $\sigma_{n}^{2}=\frac{N_{o}^{2}}{M_{s}}$.

In particular, we would like to express $P_{M D}$ in terms of sensor speed, i.e., $v_{n}$. For this, we approximate the term including $\rho_{n}$ in Eq. (11) by first substituting $e^{-v_{n} \Delta t / d_{\text {corr }}}$ into $\rho_{n}$ and then using Taylor series expansion:

$$
\sum_{n} \frac{\left(1-\rho_{n}\right) M+2 \rho_{n}}{1+\rho_{n}} \approx N+\sum_{n} \frac{\left(1-e^{-v_{n} \Delta t / d_{c o r r}}\right) M}{2} .
$$

Then, by substituting Eq. (12) into Eq. (10), we get $P_{M D}$ in terms of sensor speeds as:

$$
\begin{aligned}
& P_{M D}=1-Q\left(Q^{-1}\left(P_{F A}\right)-\frac{\left(\mu_{1}-\mu_{0}\right)}{2}\right. \\
&\left.\times \sqrt{\frac{N M}{\sigma_{n}^{2}}+\frac{M_{s}}{2 N_{o} P_{R}}\left(N+\sum_{n} \frac{\left(1-e^{\left.-v_{n} \Delta t / d_{\text {corr }}\right) M}\right.}{2}\right)}\right) .
\end{aligned}
$$

Eq. (13) indicates that $P_{M D}$ depends on the number of sensors $(N)$, number of sensings $(M)$, and more importantly, sensor speeds $\left(\left\{v_{n}\right\}_{n=1}^{N}\right)$. The impact of sensor mobility on sensing performance will be detailed in Section 5.3.

Proposition 1. PMD can be made arbitrarily small given an arbitrary $P_{F A}$ by increasing the number of times to sense.

Proof. From Eqs. (10) and (11), we have:

$$
\forall v_{n} \in \mathbb{R} \quad \lim _{M \rightarrow \infty} P_{M D}=0 \quad \text { as } \quad \lim _{M \rightarrow \infty} \sqrt{\mathbf{1}^{T} \boldsymbol{\Sigma}^{-1} \mathbf{1}}=\infty .
$$

Therefore, the proposition follows.

Remark: The authors of [5] have shown that the miss detection performance under cooperative sensing with spatiallycorrelated sensors does not converge to 0 . By contrast, Proposition 1 states that the miss detection performance of even a single mobile sensor converges to 0 . The insight behind this is that, with multiple co-located static sensors, their spatial RSS diversities are limited to their geographical area, whereas a single mobile sensor can fully exploit spatiotemporal RSS diversity as it keeps moving around the entire cell, the area of which is much larger than the correlation distance.

\subsection{Numerical Results}

Here we present numerical results of spectrum sensing performance in various wireless environments. Our on-going work includes a simulation-based study to corroborate our numerical findings.

Fig. 3(a) shows the impact of sensor speed on sensing performance. The figure indicates that as the sensor speed increases, $P_{M D}$ decreases thanks to the increased spatiotemporal diversity in measured RSSs. At a fixed speed, sensor mobility yields a better performance in urban environments due to the short decorrelation distance. 


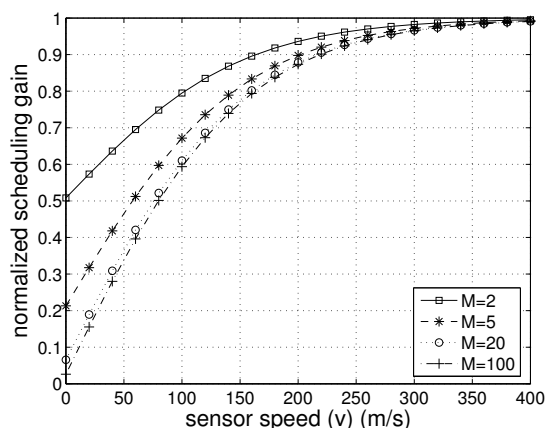

Figure 4: Sensing scheduling gain under various sensor speeds.

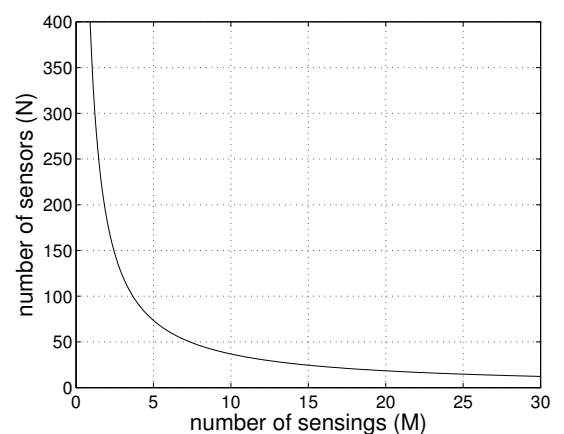

Figure 5: Combinations of $(N, M)$ to meet the detection requirements.

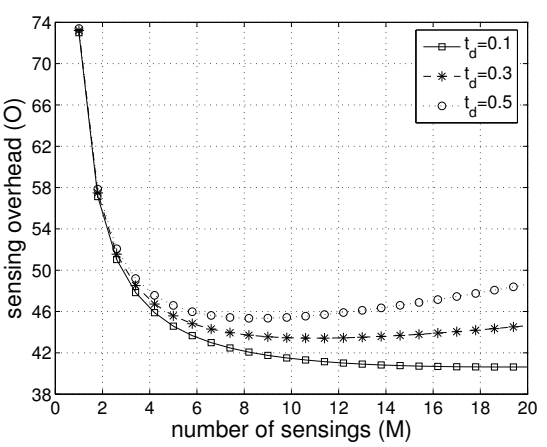

Figure 6: Minimum sensing overhead under different sensing costs.
Fig. 3(b) indicates that $P_{M D}$ decreases as the number of times to sense increases regardless of the sensor speed. Interestingly, as the sensor speed increases, the benefit from sensing scheduling increases because the correlation between consecutive measurements diminishes. This implies that, with slowly moving sensors, increasing the number of cooperating sensors might be more efficient than sensing multiple times, and vice versa (see Section 6 for details).

Fig. 3(c) plots $P_{M D}$ for various average received primary signal strengths (i.e., $\left.P_{R, 1}\right)$. As expected, $P_{M D}$ suffers from low average RSSs regardless of the sensor speed. However, sensor mobility lowers the operation range of the energy detector for the same number of measurements by better exploiting the spatio-temporal diversity in RSSs.

\subsection{Performance of Sensing Scheduling with Mobile Sensors}

We now quantify the performance gain (in terms of $P_{M D}$ for a fixed $P_{F A}$ ) of sensing scheduling with mobile sensors.

Proposition 2. (Sensing Scheduling Gain) The performance of spectrum sensing with a mobile sensor of speed $v$ sense a channel $M$ times at a discrete time interval $\Delta t$ is equivalent to having the following number of i.i.d. sensors each making a single measurement:

$$
\mathcal{G}_{\text {schedule }}=\frac{2 P_{R} M+N_{o} \frac{\left(1-\rho_{n}\right) M+2 \rho_{n}}{1+\rho_{n}}}{2 P_{R}+N_{o}},
$$

where $\rho_{n}=e^{-v_{n} \Delta t / d_{\text {corr }}}$.

Proof. Eq. (11) indicates that, with $N$ stationary sensors with a single measurement (i.e., $v_{n}=0 \forall n$ and $M=1$ ), $\rho_{n}=0 \forall n$, thus $\mathbf{1}^{T} \boldsymbol{\Sigma} \mathbf{1}=\frac{N}{\sigma_{n}^{2}}+\frac{N M_{s}}{2 N_{o} P_{R}}$. On the other hand, with a single sensor moving at speed $v_{n}>0$, it becomes $\mathbf{1}^{T} \boldsymbol{\Sigma} \mathbf{1}=\frac{M}{\sigma_{n}^{2}}+\frac{M_{s}}{2 N_{o} P_{R}} \frac{\left(1-\rho_{n}\right) M+2 \rho_{n}}{1+\rho_{n}}$. Therefore, by comparing the two cases, the proposition follows.

Remark: Proposition 2 implies that the scheduling gain increases as the sensor speed $(v)$ increases. Interestingly, in an extreme case where $v \rightarrow \infty, \rho_{n} \rightarrow 0$, thus the scheduling gain approaches $M$, i.e., scheduling sensing $M$ times is equivalent to having $M$ sensors with i.i.d. measurements. This is because when the geographical separation between consecutive sensings is greater than the decorrelation distance, the sensor will have independent measurements. Fig. 4 shows that the normalized scheduling gain converges to 1 as sensor speed increases, and at fixed sensor speed, a higher gain is achievable with a smaller number of sensings.

\section{SPECTRUM SENSING OPTIMIZATION}

In this section, we first study the tradeoff between sensor cooperation and sensing scheduling. On the basis of our findings, we then derive an optimal sensing strategy to minimize the sensing overhead.

\subsection{Cooperation vs. Scheduling}

Although both sensor cooperation and sensing scheduling are viable means to improve the sensing performance, they provide different performance gains at different sensing costs. The optimal combinations of $(N, M)$ to achieve the required detection performance can be derived from Eqs. (10) and (11) in Section 5.1.

Fig. 5 plots such possible combinations of $(N, M)$ that achieve the desired detection performance of $P_{F A}=P_{M D}=$ 0.001 with mobile sensors at $v=5(\mathrm{~m} / \mathrm{s})$. The figure shows that the number of sensors needed decreases as the number of times to sense increases, thus demonstrating the tradeoff between cooperative sensing and sensing scheduling.

\subsection{Minimization of Sensing Overhead}

As mentioned previously, sensor cooperation and sensing scheduling improve the sensing performance at different levels of degradation. Thus, our objective is to minimize the sensing overhead while meeting the detection requirements. For example, the larger the number of sensors to be used, the greater the sensing reporting time at the end of each sensing (quiet) period. This can result in a reduced time duration for data transmission, thus degrading the qualityof-service (QoS) of secondary networks. On the other hand, the more times each sensor is scheduled, the longer the delay in making a final decision on the existence of a primary signal. ${ }^{5}$ For mobile sensors, energy-efficiency is also critical to the network performance. Therefore, it is important to strike a balance between these design parameters in devising the best sensing strategy.

We consider the following sensing overheads:

- Reporting time $\left(t_{r}\right)$ : Sensors need to report their sensing results to the base station at the end of each sensing period. Thus, they must suspend data transmission during this reporting period.

- Detection delay $\left(t_{d}\right)$ : Prompt detection of a primary signal is important for the protection of primary communications. The detection delay will increase proportionally to the number of times to sense.

\footnotetext{
${ }^{5}$ Sensing must be scheduled to meet the 2-second detection
} delay requirement (i.e., CDT) in IEEE 802.22 WRANs. 
- Energy consumption $(\epsilon)$ : Energy consumption is also an important metric, especially with battery-powered mobile sensors. The total energy consumption is proportional to the total number of times to sense at cooperating sensors.

For a given set of $N$ sensors, with $M$ sensings scheduled for each sensor, the sensing overhead can be expressed as:

$$
\mathcal{O}\left(N, M, t_{r}, t_{d}, \epsilon\right)=N t_{r}+M t_{d}+M N \epsilon .
$$

Recall that our objective is to find an optimal combination of $(N, M)$ that minimizes the sensing overhead while meeting the detection requirements. It can be found as:

$$
\left(N^{*}, M^{*}\right)=\arg \min _{N, M \in \mathbb{N}} \mathcal{O}\left(N, M, t_{r}, t_{d}, \epsilon\right) .
$$

The solution of Eq. (16) can be found by taking a derivative with respect to $M$ (or $N$ ) and setting it equal to 0 .

Fig. 6 plots the total sensing overhead $(\mathcal{O})$ in terms of the number of times to sense $(M)$ to achieve the detection performance $P_{M D}=P_{F A}=0.001$ with sensor speed fixed at $v=5(\mathrm{~m} / \mathrm{s})$. Note that the corresponding optimal number of sensors $(N)$ is shown in Fig. 5. For illustrative purposes, we set the parameters as $t_{r}=0.1$ and $\epsilon=0.1$, and tried three different values for the delay cost, i.e., $t_{d} \in\{0.1,0.3,0.5\}$. The figure indicates that as the sensing delay cost $t_{d}$ increases, the number of sensings $M$ should be reduced while increasing the number of collaborating sensors $N$ in order to minimize the total sensing overhead.

\section{CONCLUDING REMARKS}

Numerous cooperative sensing algorithms in CRNs have been proposed, but none of them exploits the advantages of mobile sensors. In this paper, we study the impact of sensor mobility on the performance of spectrum sensing in a joint sensor cooperation and sensing scheduling framework. Our theoretical analysis confirms the intuition that sensor mobility increases spatio-temporal diversity in RSSs, thus improving the sensing performance. The analytical results indicate that the sensing scheduling gain increases with fast moving sensors, leading to an interesting tradeoff between sensor cooperation and sensing scheduling. We exploit this tradeoff to find the optimal combination of the number of sensors for cooperation and the number of times to sense so as to minimize the sensing overhead.

The work presented in this paper is an important step toward consideration of sensor mobility in CRNs, and opens up an interesting new approach to spectrum sensing in CRNs. In future, we will investigate how to leverage sensor mobility for detection of small-scale primary users, e.g., wireless microphones. It would also be interesting to study how to use a hybrid of static and mobile sensors to maximize the sensing performance.

\section{Acknowledgments}

The work reported here is supported in part by the NSF under grants CNS-0519498 and CNS-0721529.

\section{REFERENCES}

[1] A. Algans, K. I. Pedersen, and P. E. Mogensen. Experimental Analysis of the Joint Statistical Properties of Azimuth Spread, Delay Spread, and Shadow Fading. IEEE Journal on Selected Areas in Communications, 20(3):523-531, April 2002.
[2] D. Cavalcanti and C. Cordeiro. Proposed Resolution for Comments Related to the Superframe and Sensing. IEEE 802.22-07/0176r0, April 2007.

[3] K. R. Chowdhury, M. D. Felice, and I. F. Akyildiz. TP-CRAHN: A Transport Protocol for Cognitive Radio Ad-hoc Networks. In Proc. IEEE INFOCOM '09, pages 2482-2490, April 2009.

[4] C. Cordeiro, K. Challapali, D. Birru, and S. Shankar. IEEE 802.22: An Introduction to the First Wireless Standard based on Cognitive Radio. Journal of Communications, 1(1):38-47, April 2006.

[5] A. Ghasemi and E. S. Sousa. Asymptotic Performance of Collaborative Spectrum Sensing under Correlated Log-Normal Shadowing. IEEE Communications Letter, 11(1):34-36, Jan 2007.

[6] M. Grossglauser and D. N. C. Tse. Mobility Increases the Capacity of Ad Hoc Wireless Networks. IEEE/ACM Transactions on Networking, 10(4):477-486, Aug 2002.

[7] M. Gudmundson. A Correlation Model for Shadow Fading in Mobile Radio. Electronic Letters, 27(23):2146-2147, Nov 1991.

[8] Y.-C. Liang, Y. Zeng, E. C. Y. Peh, and A. T. Hoang. Sensing-Throughput Tradeoff for Cognitive Radio Networks. IEEE Transactions on Wireless Communications, 7:1326-1337, 2008.

[9] B. Liu, P. Brass, O. Dousse, P. Nain, and D. Towsley. Mobility Improves Coverage of Sensor Networks. In Proc. ACM MobiHoc '05, pages 300-308, May 2005.

[10] J. Luo and J.-P. Hubaux. Joint Mobility and Routing for Lifetime Elongation in Wireless Sensor Networks. In Proc. IEEE INFOCOM '05, pages 1735-1746, Mar 2005.

[11] A. W. Min and K. G. Shin. An Optimal Sensing Framework Based on Spatial RSS-profile in Cognitive Radio Networks. In Proc. IEEE SECON '09, June 2009.

[12] S. M. Mishra, A. Sahai, and R. W. Brodersen. Cooperative Sensing among Cognitive Radios. In Proc. IEEE ICC 'O6, pages 1658-1663, June 2006.

[13] H. V. Poor. An Introduction to Signal Detection and Estimation. 2nd ed. New York: Springer-Verlag, 1994.

[14] P. Samar and S. B. Wicker. On the Behavior of Communication Links of a Node in a Multi-Hop Mobile Environment. In Proc. ACM MobiHoc '04, pages 145-156, May 2004.

[15] Y. Selén, H. Tullberg, and J. Kronander. Sensor Selection for Cooperative Spectrum Sensing. In Proc. IEEE DYSPAN' '08, Oct 2008.

[16] S. Shellhammer, S. Shankar, R. Tandra, and J. Tomcik. Performance of Power Detector Sensors of DTV Signals in IEEE 802.22 WRANs. In Proc. ACM TAPAS '06, Aug 2006.

[17] A. Taherpour, Y. Norouzi, M. Nasiri-Kenari, A. Jamshidi, and Z. Z. Yazdi. Asymptotically Optimum Detection of Primary User in Cognitive Radio Networks. IET Communications, 1(6):1138-1145, Dec 2007.

[18] V. Tawil et al. DRAFT 802.22a (amendment) PAR. IEEE 802.22-09/0029r0, Jan 2009.

[19] E. Visotsky, S. Kuffner, and R. Peterson. On Collaborative Detection of TV Transmissions in Support of Dynamic Spectrum Sharing. In Proc. IEEE DySPAN '05, pages 338-345, Nov 2005. 